Revista Iberoamericana de Ciencias de la Actividad Física y el Deporte

\title{
PLAN PREVENTIVO DE ISQUIOTIBIALES EN FUTBOLISTAS DE CATEGORÍA INFANTIL
}

PREVENTIVE PLAN HAMSTRING IN FOOTBALL KIDS

\section{Pinazo Hilario, A. ; García-Revillo Muñoz, J.J. ${ }^{2 ;}$ y Guerrero Calderón, A. ${ }^{3}$}

${ }^{1}$ Máster de readaptación físico-deportiva (Medac, Málaga), Licenciado en CCAFD, Granada (España), Diplomado en Magisterio Educación Física, Málaga (España) adrian_pinazohilario@hotmail.com

2 Máster de readaptación físico-deportiva (Medac, Málaga), Licenciado en CCAFD, Sevilla (España), Diplomado en Magisterio Educación Física, Málaga (España) Juanjo1990pf@gmail.com

3 Máster de readaptación físico-deportiva (Medac, Málaga), Licenciado en fisioterapia, Barcelona (España) aguerrero.calderon@gmail.com

Código UNESCO: 3212 Salud Pública

Clasificación Consejo de Europa: 17. Otras (Educación Física y Salud)

Recibido el 14 de diciembre de 2014

Aceptado el 22 de junio de 2015

Correspondencia:

Adrián Pinazo Hilario: adrian_pinazohilario@hotmail.com

\section{RESUMEN}

El propósito de nuestro estudio es trabajar en un programa de prevención de lesiones en isquiotibiales en jóvenes futbolistas de la etapa infantil (13-14 años), realizando un pretest a todos los sujetos (4 grupos), aplicando un entrenamiento de ADM isquiotibial diferente, y volviendo a hacer un posterior postest transcurridas 10 semanas de entrenamientos, para comprobar qué grupo mejora más trasladando esas mejoras al entrenamiento específico.

Para llevar a cabo nuestro estudio hemos requerido 44 jugadores de la categoría infantil. La muestra se clasificó por grupo trabajo por pareja $(n 1=13)$, trabajo en parejas + trabajo excéntrico (Hamstrings Nordics) $(\mathrm{n} 2=10)$, trabajo SGA (n3=7) y trabajo SGA + trabajo excéntrico (Hamstrings Nordics) (n4=14), siendo cada grupo un equipo diferente. Los resultados obtenidos de este 
estudio son que con cualquier tipo de entrenamiento de ADM vamos a mejorar esta cualidad, sin grandes diferencias entre los diferentes grupos.

\section{PALABRAS CLAVE}

Amplitud de Movimiento, excéntrico, infantil, isquiotibial, prevención

\section{ABSTRACT}

The purpose of our study is to work out about a program of hamstring injury prevention's in young footballers in his infant stage (13-14 years),

At the beginning, we performing a pretest for all subjects (4 groups) to have information to compare in the end, then we stablished different types of training hamstring ADM to returning to make a subsequent posttest beyond 10 weeks of training, to can compare which group have a better improvement and used it according to a specific training in football.

To carry out our study we required 44 players in the junior class. The sample have been classified by group work in couple $(\mathrm{n} 1=13)$, working in pairs + eccentric work (Hamstrings Nordics) (n2 = 10), SGA (n3=7) labor and SGA + eccentric work (Hamstrings Nordics) work ( $\mathrm{n} 4=14$ ), each group belonging in a different team. As a results of this study, we have to hightlight that any type of training ADM will improve this quality, without significant differences between groups.

\section{KEY WORDS}

Range of motion (ROM), eccentric, kids, hamstring, football soccer, prevention. 


\section{INTRODUCCIÓN}

Hoy día las lesiones en los isquiotibiales es la lesión muscular más frecuente en el fútbol, como podemos ver en el gráfico siguiente proporcionado por la UEFA.

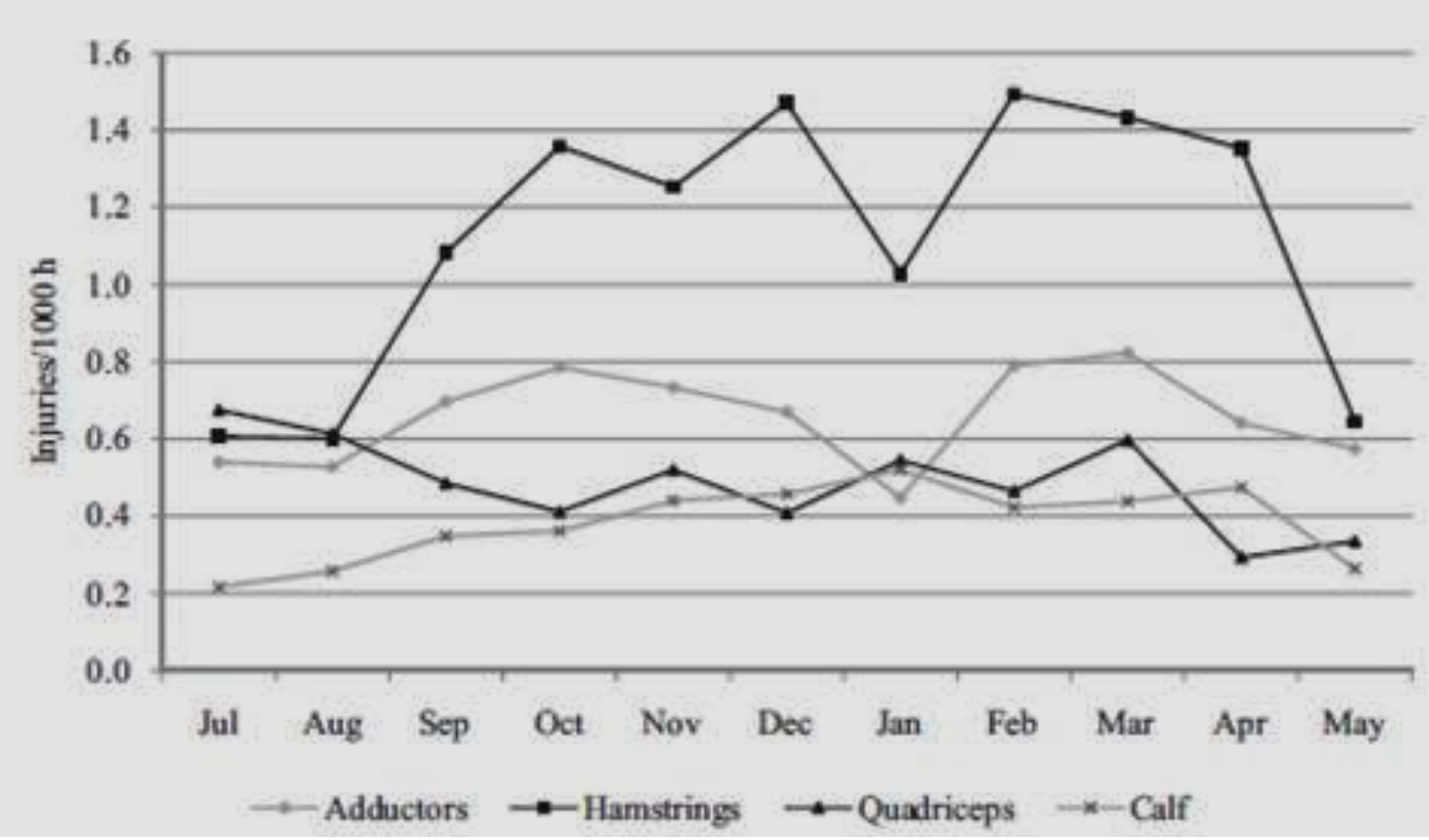

Figura 1 : Lesiones musculares a lo largo del año por cada 1000h de práctica. The UEFA injury study (Hägglund, Walden \& Ekstrand, 2012)

Schmitt B. et al. (2013) y Heiderscheit B. (2010) siguen la misma línea que el informe UEFA, estando el índice lesional entre el $12-16 \%$ en el caso del primero y afirmando que supone un porcentaje sustancial en el caso del segundo. En el caso de Roberts S. (2013) llegó a ser de hasta el 56\%. Melegati G. (2014) también encontró que los isquiotibiales son la musculatura con más índice de lesión ( $n=11$ de $n=20$, donde 20 es el número total de lesiones musculares y 11 de esas 20 de isquiotibial).

Además, es una lesión que una vez que se ha producido tiene un alto porcentaje de recidivas, como muestra el estudio de De Visser et al. (2012), que realizando una revisión bibliográfica sobre la literatura de este tema, muestran que hay un porcentaje entre el 13,9 y el $63,3 \%$ de volver a recaer de esa lesión en los dos años posteriores a la primera lesión en la zona. Sanfilippo 
et al. (2013) muestran un porcentaje por encima del 30\% de recaídas en las dos primeras semanas del retorno a jugar.

Debido a estos números tan elevados, habrá que abordar el tratamiento de la lesión desde la prevención, ya que si conseguimos reducir el minimizar el riesgo de lesión, minimizamos un buen porcentaje de lesión, ya que como ya se ha comentado, hay una alta probabilidad de recaídas, como McCall A (2014) que muestra que el mayor riesgo de lesión de isquiotibial es haberla tenido.

En muchas ocasiones nos centramos en la prevención en edades ya competitivas, olvidándonos de las etapas de formación, más importantes quizás, ya que cimientan una base del futuro trabajo.

Por tanto, tendremos que ver y analizar los factores de riesgo, siendo uno de ellos un excesivo estiramiento durante el sprint o el salto (Cohen et al. 2011), por lo que una de nuestras intensiones es minimizar este factor, ya que el fútbol por su naturaleza tiende a acortar esta musculatura. Czaprowski et al. (2013) sigue esta línea de flexibilidad reducida de isquiotibiales en adolescentes, comparando tres programas. A pesar de esta importancia, la ADM, es la cualidad física olvidada por excelencia por parte de los docentes en Educación Física, entrenadores y monitores deportivos (Ramos D. et al. 2007), aunque sí que es cierto que la tendencia está cambiando en los últimos años. En el congreso de Pontevedra, llevado a cabo por Muyor J. et al. (2012) se pone de manifiesto que la disminución de la extensibilidad isquiosural conlleva un mayor riesgo de lesión.

¿Por qué trabajo en fase excéntrica? Otro factor lesional es la fase excéntrica en la fase terminal de vuelo, por lo que es un foco de atención para ejercicios en altas cargas (Alonso et al, 2012), en la misma línea que Padulo J. (2013) donde comenta que la fase de desaceleración y de aterrizaje son las críticas. Sanfilippo et al. (2013) añaden como hay un $22 \%$ de déficits de fuerza excéntrica en jugadores salidos de la lesión. Sugiura et al. (2008) mostraron como 6 sprinter que terminaron con una lesión, tenían picos de fuerza 
excéntrica inferiores. de Malliaropoulos et al. (2013) desarrollan un programa de trabajo excéntrico, justificándolo con el mayor aumento de la masa, fuerza y poder del isquiotibial, que un entrenamiento concéntrico. En un estudio de EMG se mostró como hubo una pérdida de fuerza excéntrica en el bíceps femoral después de realizar algún sprint Timmins R. (2014). Por ello, con nuestro trabajo queremos comprobar si en edades tempranas el trabajo de stretching y entrenamiento excéntrico nos podría reportar ventajas para la prevención. Yeung et al. (2009) y Tol J. (2014) muestran un déficit en el ratio excéntrico isquiotibial/concéntrico de cuádriceps. Otra evidencia más se recoge en el estudio de Brukner P. et al. (2013) en el cual el programa de fuerza excéntrico tuvo una genial respuesta. En este mismo trabajo, se pone de manifiesto que las lesiones de isquiotibiales muestran una reducida fuerza excéntrica en esta musculatura. Por último, Schmitt B. et al. (2004) describen las incidencias lesionales de este músculos, siendo todas ellas en situaciones de régimen excéntrico. Por todas estas evidencias, hemos querido proponer el trabajo excéntrico en dos de nuestros programas. En el mismo estudio de Schmitt B. et al. (2004) comentan que los autores sienten que el trabajo excéntrico debería hacerse no solo en las posiciones de sedestación, si no que debería incluirse ejercicios en posiciones de alargamiento. Nosotros ante la dificultad de llevar a cabo la segunda opción, creemos que aunque no se trabaje de ambas formas, sí que ayudaría a desplazar la curva de fuerza.

Uno de los test que vamos a utilizar para evaluar la ADM isquiotibial con el test Sit and reach, test que comienza a utilizarse en la década de los 50 (Arregui, JA, et al. 2001). En Fernández, E. et al. (2011) nos dice que el test Sit and Reach es el test lineal más utilizado (López et al. 2008). En el mismo artículo de Fernández, E. et al. comenta que también es el más usado en ambientes escolares, ya que su realización es simple, fácil de administrar y requiere un mínimo entrenamiento (Rodríguez et al. 2008).

También en el estudio de Arregui JA (2001) nos muestra que Leighton comienza en los años 60 a hacer diferentes estudios sobre esta cualidad, poniéndose ya de manifiesto cierta dificultad para medir esta cualidad, además 
de mostrarse que los jóvenes activos tienen números mayores que los sedentarios. El test usado por nosotros, tiene según Palma et al. (2010) cierta controversia en su evaluación, habiendo otros estudios que lo apoyan llegando a tener un $90 \%$ de fiabilidad según Nupponen (1981). Alguno más reciente como es el caso de López P. et al. (2007) nos comenta que muchos estudios sobre la validez y fiabilidad de los test Sit and Reach demuestran una validez de moderada a alta para la flexibilidad isquiotibial $(0,39-0,89)$.

En los últimos años Merino et al. (2010) sugieren que un acortamiento de gemelos puede afectar en los registros que se puedan alcanzar en la prueba. Antes Di Santo (2000) también habla de la importancia tanto de gemelos como de lumbares en el test.

Quintasi Villarroel et al. (2006), realizan un continuum de resultados estandarizados del test Sit and Reach sobre una población escolar. Aunque nosotros no vamos a estandarizar los resultados, si no que veremos la evolución de cada jugador.

Tabla 1: Relación Condición Física y Rendimiento del Test de Sit and Reach

\begin{tabular}{|c|c|}
\hline Condición Física & Rendimiento (cm) \\
\hline Buena & $20-15$ \\
\hline Aceptable & $14-10$ \\
\hline Baja & $09-05$ \\
\hline Deficiente & $04-00$ \\
\hline Problemas de ADM & Inferior 00 \\
\hline
\end{tabular}

Otra de las pruebas llevadas a cabo es el test dedos-suelos, ya que como comenta López P. et al. (2010) sería interesante realizar una medición en bipedestación ya que es una de las posiciones habituales en la vida cotidiana.

Por otro lado, nosotros queremos ver la correlación que pudieran tener el entrenamiento de la ADM en dicha musculatura en la mejora de la fuerza en el tren inferior, que será medida con un test de salto CMJ. Hedrick A. (2004) comenta que en tenistas un mayor rango de movimiento en la articulación del hombro, lleva a una mayor potencia en el saque. 
Por último, dos de nuestros grupos han utilizado el Nordic hamstring como trabajo excéntrico, ya recomendado en otros estudios como es el de Daneshjoo A. (2013).

En definitiva, según Daneshjoo A. (2013) (Sander et al. 2013) habría que enfocar el trabajo preventivo hacia los mecanismo que propician la posible lesión, incrementar la temperatura en el cuerpo, aumentar el riego sanguíneo a los músculos, y a lo que nosotros nos centramos, aumentar elasticidad de los músculos y actividad neural, lo que es definida por Sale (2002) como un incremento en la eficiencia del músculo para producir fuerza después del calentamiento.

\section{OBJETIVOS}

Los objetivos del estudio son los siguientes:

- Determinar el grado de ganancia de cada forma de trabajo de la musculatura isquiotibial.

- Relacionar la ADM con otras cualidades físicas.

- Conocer qué modo de trabajo de esa musculatura nos puede hacer prevenir una mayor incidencia lesional.

\section{MATERIAL Y MÉTODO}

\section{Sujetos}

El estudio ha sido realizado en la ciudad de Málaga (España), siendo la población referencia jóvenes futbolistas de categoría infantil (fecha de nacimiento 2000-2001). La muestra utilizada (13-14 años, 44 chicos) procede de 2 clubes de la provincia.

La muestra se compone de 44 jugadores de categoría infantil, los cuales fueron agrupados en 4 grupos diferentes, trabajo por parejas $\left(n_{1}=13\right)$, trabajo por parejas + trabajo excéntrico (Hamstrings Nordics) $\left(n_{2}=10\right)$, trabajo SGA $\left(n_{3}=7\right)$ 
y trabajo SGA + trabajo excéntrico (Hamstrings Nordics) $\left(n_{4}=14\right)$, es decir, la muestra la compondrán 15 chicos determinados al azar dentro de cada equipo.

En un principio tomamos una muestra de 60 jugadores, 15 sujetos para cada grupo, llegando a pasar el test a cada uno de ellos. Pero hemos tenido que reducirla, ya sea porque están lesionados en algunos casos, o han cambiado de club en otros. La muestra inicial era de 60 jugadores previniendo que se pudieran caer cierto número de jugadores, como ha sido finalmente.

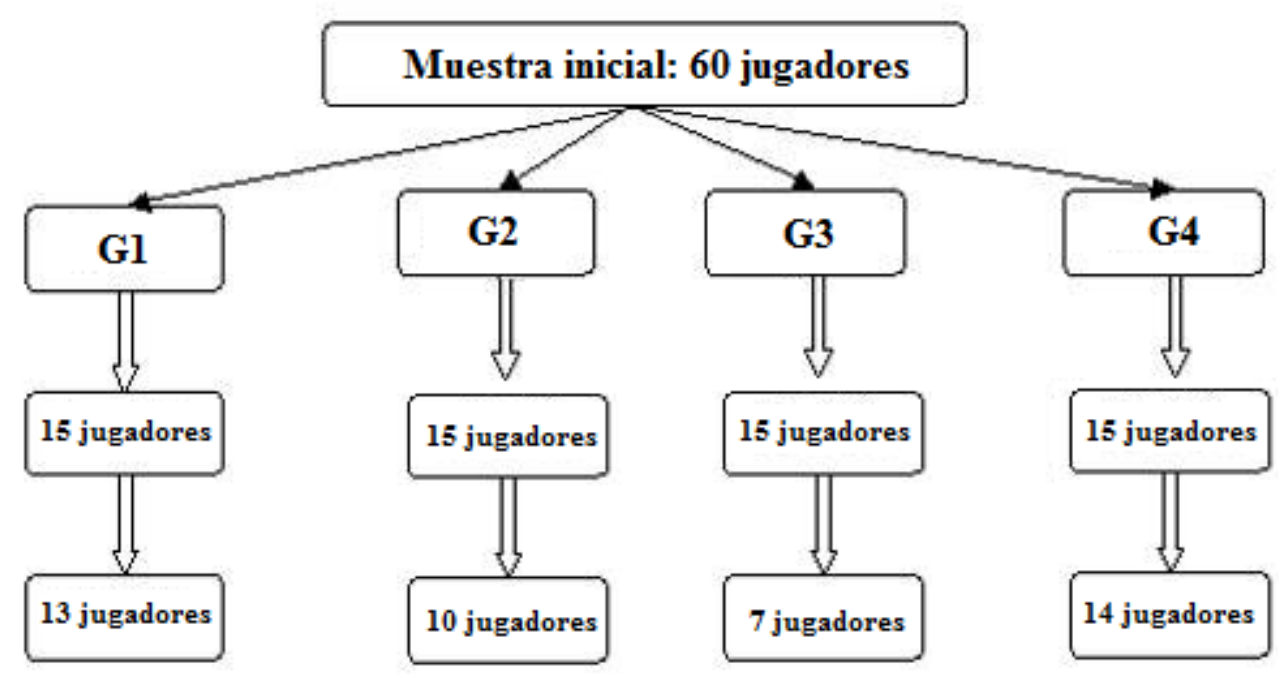

Figura 2: Esquema de la relación de jugadores que terminaron el estudio

Decir, que en los grupos en los cuales ha habido un mayor número de pérdidas ha sido en los de categoría inferior, pudiéndose deber a haber salido a realizar pruebas a otros clubes, ya que en esa fecha comienzan a realizarse los equipos del año próximo.

\section{Diseño}

El presente estudio es un diseño experimental longitudinal, en el cual hay 2 tomas de datos de todos los sujetos, una toma inicial llamada pretest y una toma final o postest. 
Hay 4 niveles de variable independiente (VI), los cuales serían cada uno de los grupos de entrenamientos ya citados. Las VI serían: trabajo por pareja, trabajo en parejas + trabajo excéntrico (Hamstrings Nordics), trabajo SGA y trabajo SGA + trabajo excéntrico (Hamstrings Nordics). Como vemos es un diseño multigrupos, para comprobar con los postest realizados a cada uno de ellos si hubieran diferencias entre cada uno de ellos, por lo que la toma de datos han sido en condiciones idénticas.

Evidentemente, hay una serie de condiciones que podrían afectar a nuestra recogida de datos, más inclusive trabajando con este tipo de población. Para ello se ha llevado a cabo lo siguiente:

- Todos los sujetos realizarán las pruebas habiendo realizado el mismo calentamiento.

- Se lleva a cabo en un tramo de hora similar, entre las 17:00 y las 19:00.

- Cada grupo será medido por el mismo evaluador y en el mismo lugar.

- Las condiciones climatológicas sería una posible variante contaminante, pero por fortuna la climatología fue idéntica en todos los test realizados.

- Por último, sí que hay una variable que nos puede afectar, y es el nivel motivacional con el que el chico pueda venir.

\section{Aparato y/o material}

Para la aplicación de los test se han requerido los siguientes recursos materiales:

1. Cajón de flexibilidad, Sit and Rich.

2. Esterilla.

3. Metro.

4. Cinta métrica.

5. Picómetro.

6. Tanita.

7. Cronómetro.

8. Goniómetro. 
9. Plataforma de salto (Chronojump)

10. Ordenador portátil.

En el test de fuerza CMJ en la plataforma, se utilizó la fórmula de Sayers (1999), ya que como dice en el estudio de Lara A. (Medición directa de la potencia con test de salto en voleibol femenino), es una fórmula que estima de forma muy cercana.

\section{Procedimiento}

Los jugadores fueron informados previamente sobre el procedimiento que iba a llevar el estudio, así como sus padres, madres o tutor legal, los cuales debieron rellenar un consentimiento firmado, el cual informaba de todo el proceso. El protocolo seguido en este estudio fue el mismo para todos los sujetos de los diferentes equipos, tanto en el pretest como el postest:

1. Vestir ropa cómoda y que no reduzca ningún tipo de movimiento.

2. Realizar todas las pruebas previamente al entrenamiento, con la única salvedad de la velocidad-agilidad, la cual se llevaba a cabo tras una rutina de calentamiento.

3. Cada prueba es recogida por la misma persona, de modo que la forma de tomar los datos sean las mismas siempre.

4. En base a los datos recogidos, realizar una tabla de valoración.

Intentando eliminar al máximo alguna variable contaminante, los diferentes test se llevaron a cabo a la misma hora y el mismo día de la semana a cada equipo, evitando distorsiones en cuanto a la graduación de la intensidad del entrenamiento por parte del entrenador.

\section{RESULTADOS}

Los resultados registrados en el pretest y postest fueron estadísticamente analizadas por el programa estadístico SPSS. A continuación, se adjunta los resultados obtenidos en las variables estudiadas: 
Tabla 2: Tabla comparación pretest-postest (medias)

\begin{tabular}{|c|c|c|c|c|c|}
\hline \multicolumn{2}{|c|}{ VARIABLES } & $\begin{array}{c}\text { Trabajo por } \\
\text { parejas }\end{array}$ & $\begin{array}{c}\text { Trabajo por } \\
\text { parejas y } \\
\text { trabajo } \\
\text { excéntrico }\end{array}$ & SGA & $\begin{array}{c}\text { SGA y trabajo } \\
\text { excéntrico }\end{array}$ \\
\hline \multirow{16}{*}{ 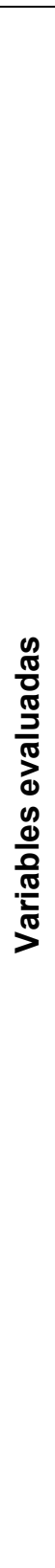 } & Categoría & $\begin{array}{c}\text { Infantil } \\
\text { autonómico }\end{array}$ & $2^{a}$ infantil & $2^{a}$ infantil & $1^{a}$ infantil \\
\hline & Talla pretest & $170 \mathrm{~cm}$ & $161,4 \mathrm{~cm}$ & $151,9 \mathrm{~cm}$ & $154,4 \mathrm{~cm}$ \\
\hline & Talla postest & $171,3 \mathrm{~cm}$ & $161,9 \mathrm{~cm}$ & $154,5 \mathrm{~cm}$ & $155,5 \mathrm{~cm}$ \\
\hline & Peso pretest & $62,6 \mathrm{Kg}$ & $52,490 \mathrm{Kg}$ & $53,7 \mathrm{~kg}$ & $44,4 \mathrm{~kg}$ \\
\hline & Peso postest & $63,3 \mathrm{Kg}$ & $54,370 \mathrm{Kg}$ & $53,7 \mathrm{Kg}$ & $46 \mathrm{~kg}$ \\
\hline & IMC pretest & $15,5 \%$ & $16,03 \%$ & $21,3 \%$ & $12,7 \%$ \\
\hline & IMC postest & $13,8 \%$ & $15,43 \%$ & $18,6 \%$ & $12,4 \%$ \\
\hline & $\begin{array}{l}\text { Valores medios sit } \\
\text { and reach pretest }\end{array}$ & $15,6 \mathrm{~cm}$ & $15,05 \mathrm{~cm}$ & $18,4 \mathrm{~cm}$ & $14 \mathrm{~cm}$ \\
\hline & $\begin{array}{l}\text { Valores medios sit } \\
\text { and reach postest }\end{array}$ & $17,6 \mathrm{~cm}$ & $15,3 \mathrm{~cm}$ & $19,7 \mathrm{~cm}$ & $15,4 \mathrm{~cm}$ \\
\hline & $\begin{array}{l}\text { Valores medios sit } \\
\text { and reach de mejora }\end{array}$ & $2 \mathrm{~cm}$ & $0,25 \mathrm{~cm}$ & $1,3 \mathrm{~cm}$ & $1,6 \mathrm{~cm}$ \\
\hline & $\begin{array}{l}\text { Valores medios test } \\
\text { dedos-suelo pretest }\end{array}$ & $0 \mathrm{~cm}$ & $-0,9 \mathrm{~cm}$ & $2,7 \mathrm{~cm}$ & $-1,7 \mathrm{~cm}$ \\
\hline & $\begin{array}{l}\text { Valores medios test } \\
\text { dedos-suelo postest }\end{array}$ & $0,9 \mathrm{~cm}$ & $1,4 \mathrm{~cm}$ & $3,3 \mathrm{~cm}$ & $-0,4 \mathrm{~cm}$ \\
\hline & $\begin{array}{c}\text { Valores medios test } \\
\text { dedos-suelo de } \\
\text { mejora }\end{array}$ & $0,9 \mathrm{~cm}$ & $2,5 \mathrm{~cm}$ & $0,6 \mathrm{~cm}$ & $1,3 \mathrm{~cm}$ \\
\hline & $\begin{array}{c}\text { Valores medios CMJ } \\
\text { pretest }\end{array}$ & $\begin{array}{c}2642,386 \\
W\end{array}$ & $1818,115 \mathrm{~W}$ & $1686,907 \mathrm{~W}$ & 1668,286 W \\
\hline & $\begin{array}{c}\text { Valores medios CMJ } \\
\text { postest }\end{array}$ & $2606,51 \mathrm{~W}$ & $1682,717 \mathrm{~W}$ & $1745,455 \mathrm{~W}$ & $1632,922 \mathrm{~W}$ \\
\hline & $\begin{array}{c}\text { Valores medios CMJ } \\
\text { de mejora }\end{array}$ & $-35,875 \mathrm{~W}$ & $-135,397 \mathrm{~W}$ & $58,548 \mathrm{~W}$ & $-35,364 \mathrm{~W}$ \\
\hline
\end{tabular}

A continuación se adjuntan los gráficos de las mejoras recogidas en las pruebas de forma individual, donde se verá más claramente qué grupo ha evolucionado más, aunque realmente las diferencias no han sido demasiado significativas. 


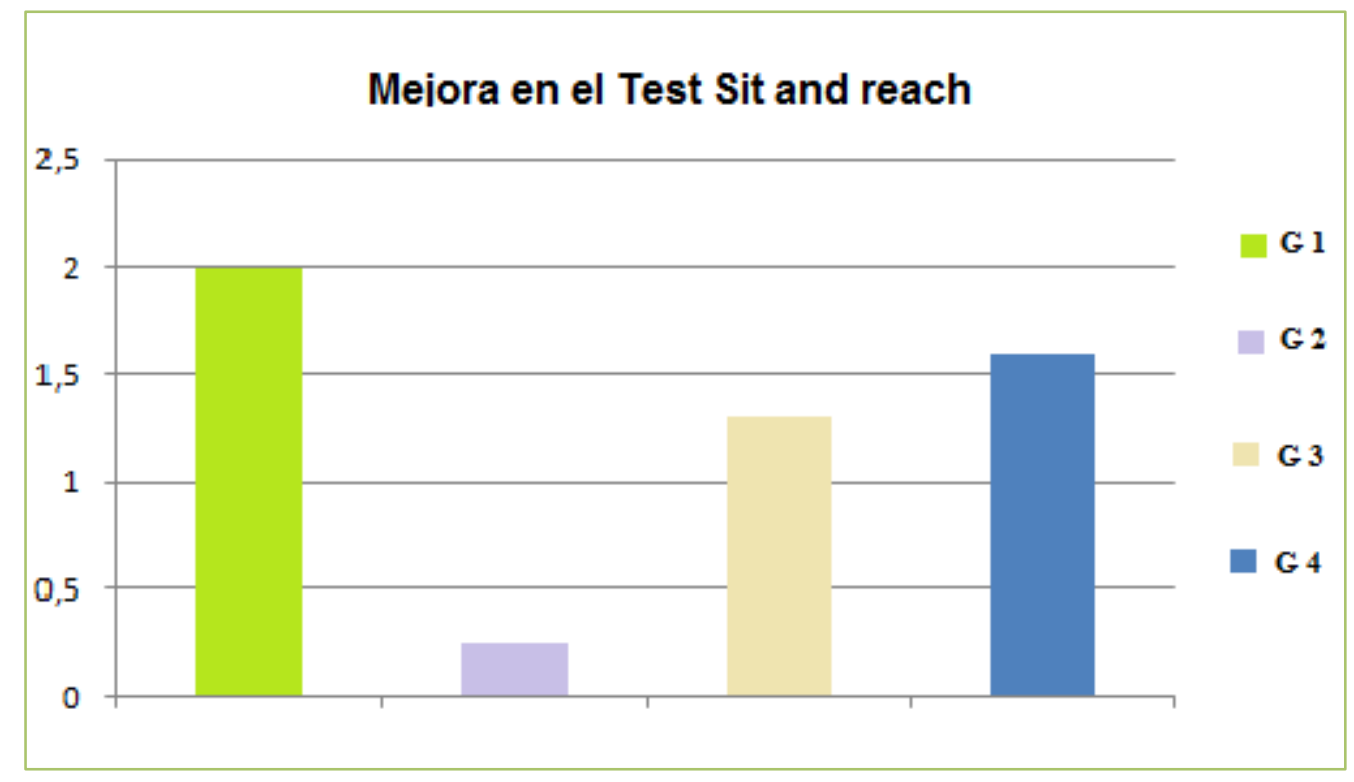

Gráfica 1: Mejora en el test Sit and Reach (cm)

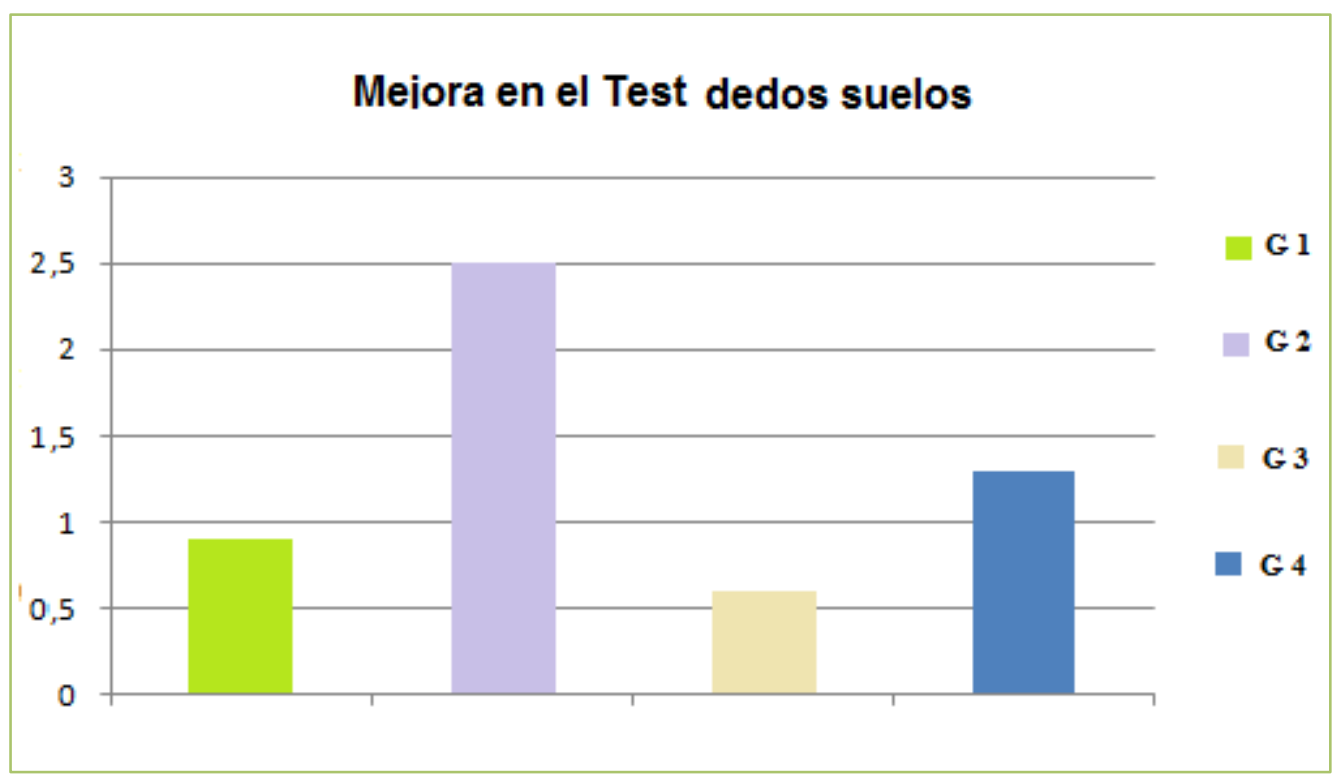

Gráfica 2: Mejoras obtenidas en el test dedos suelos (cm) 


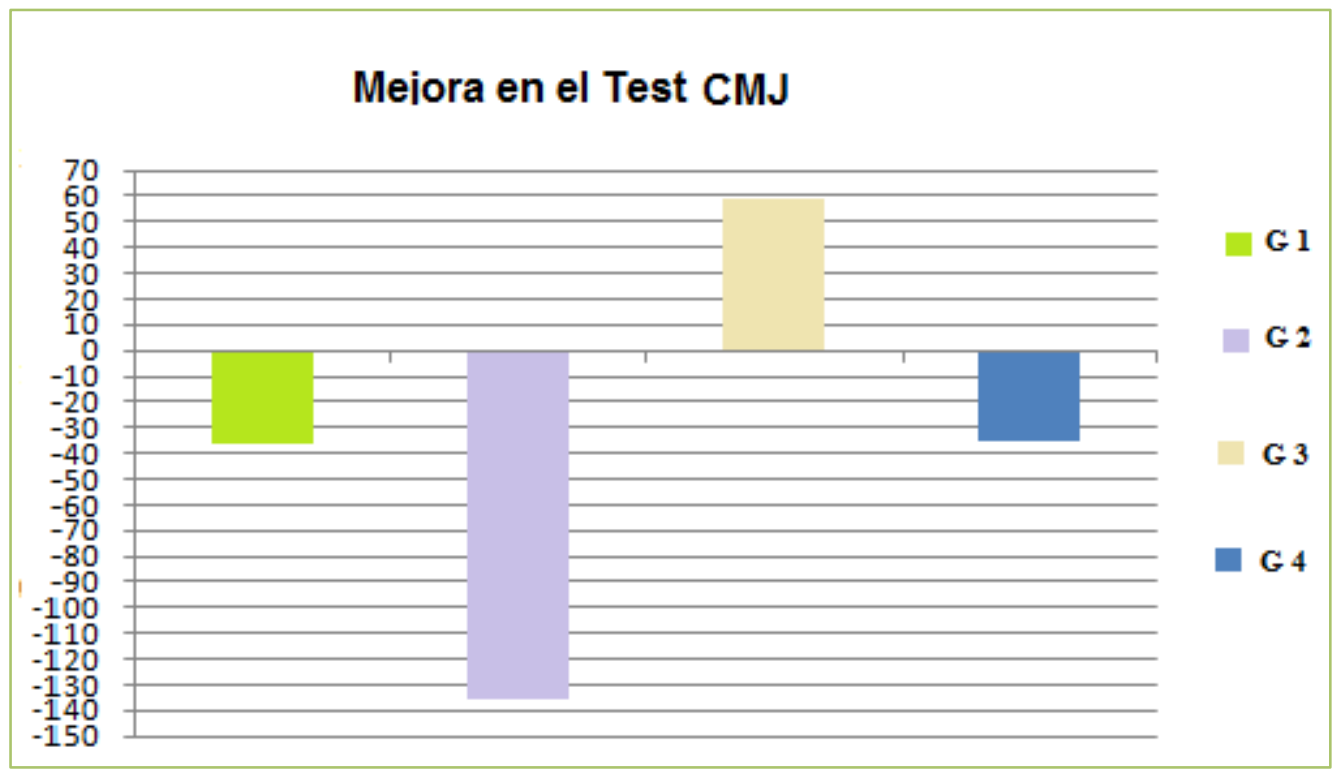

Gráfica 3: Mejoras obtenidas en el CMJ (ms)

Como ya hemos comentado unos párrafos antes, los resultados que se han obtenidos no han sido demasiado significativo, pudiendo deberse a que el tiempo de entrenamiento no ha sido el suficiente como para mostrar unas diferencias claras e indicar el mejor plan de trabajo para implantarlo como prevención, por lo que con estos resultados, realizando un trabajo específico para ampliar la ADM puede ayudarnos.

Sí que es cierto que en el test dedos-suelos los resultados obtenido en los grupos que llevaron a cabo trabajo excéntrico son algo más superiores, por lo que puede servirnos de guía para utilizarlo en nuestros entrenamientos.

\section{DISCUSIÓN}

Una vez recogido los datos del estudio, reflejan como realizando cualquier tipo de trabajo específico de ADM para la musculatura del foco de estudio, se producen ganancias medias en los grupos estudiados, sin ser relativamente significativos en el tiempo que se llevó a cabo el registro, 10 semanas. En los grupos que trabajan con ejercicios excéntricos tienen unos valores levemente superiores en el test dedos suelos, por lo que pensamos que una futura línea de actuación podría ir orientada a un entrenamiento de un número mayor de 
semanas, para analizar si hubiera una diferencia más significativa de las encontradas en este tramo de tiempo.

Si según Czaprowski et al. (2013) una flexibilidad reducida aumenta el riesgo de lesión, nos ceñimos a que tiene que haber una mayor flexibilidad para prevenir lesiones en el isquiotibial, cualquier forma de entrenamiento nos sería de ayuda para ir teniendo ganancias, al menos hasta las 10 semanas.

Siguiendo este estudio de 6 semanas de Czaprowski et al. (2013), consiguieron resultados significativamente superiores en los diferentes test realizados con un entrenamiento previo de ejercicios de estabilización combinado con estiramientos pasivos y de ejercicios post-isométricos relajación. Mientras que no hubo mejoras en el grupo de ejercicios de estabilización. Por lo que nos lleva a la conclusión, que estos últimos por sí solos no nos van a conseguir las ganancias de flexibilidad buscadas para la prevención de lesiones en isquiotibial.

Como ya se ha dicho, en nuestro estudio no hay diferencias significativas entre los 4 grupos. Creemos que puede tener alguna relación el tramo de temporada de cada grupo, ya que cuando se llevó a cabo el post test, alguno de los grupos ya habían concluido los entrenamientos, por lo que el nivel de exigencia en cuanto a entrenamientos es menor que el resto, lo que pudo hacer asemejar las diferentes variables, en caso que pudiera haber grandes diferencias.

Esto último creemos que tiene gran importancia en los niveles de potencia (W) que consiguieron los grupos que habían finalizado la temporada, ya que siendo categorías superiores, y por consiguiente el nivel de aptitud de los chicos a priori es superior, consiguieron resultados negativos medios $(-35,875 \mathrm{~W}$ grupo 1, -135,397 W grupo 2, - 35, $364 \mathrm{~W}$ grupo 4). Mientras que hay una mejora no significativa en el grupo 4 (58,548 W). Hedrick A. (2004) consiguió unos valores de fuerza incrementados en el tren superior, no pudiendo demostrar que en el tren inferior haya mejoras significativas de fuerza. 
Los valores de fuerza negativas se podrían deber a que estamos en el tramo final de liga, bajando la calidad e intensidad de los entrenos en dichas categorías, así como que los jugadores tengan fatiga muscular, ocasionando empeorar los registros.

\section{CONCLUSIONES}

Realizar cualquier tipo de entrenamiento enfocado a la mejora de ADM de isquiotibial, va a tener beneficios referente a esta cualidad, sin muchas diferencias significativas en entrenamiento de 10 semanas.

Según los valores de Quintasi Villarroel et al. (2006) la mayoría de chicos comenzaron con valores bajos-aceptables, poniéndose de manifiesto que hoy día la ADM es una cualidad olvidada por los docentes y entrenadores (Ramos D. et al. 2007).

No se ven muestras de mejorar la fuerza con el trabajo paralelo de ADM, para corroborar si hay influencias, tanto positiva, como negativa si fuera el caso, debería enfocarse el trabajo en otra época de temporada.

Por tanto, se debería enfocar una parte del entrenamiento, siguiendo nuestro estudio al final de la sesión, a llevar a cabo estiramientos, ya sean asistidos, SGA... y añadir un trabajo de ejercicios excéntricos, ya que estos últimos ya se ha mostrado eficaz en otros estudios, aumentando la fuerza en el isquiotibial (Daneshjoo A. 2013), incluso en alguno de los estudios con jugadores amateurs hay un $70 \%$ de probabilidad de reducir el riesgo de lesión (Van der Horst N. 2013).

En definitiva, si en los entrenamientos aplicamos estiramientos y trabajo excéntrico podríamos utilizarlo como modo de prevención en este tipo de lesiones, aunque como dice Rogan S. (2013) una futura línea de investigación 
debería ser si realmente los estiramientos estáticos reducen el riesgo de lesión en futbolistas.

\section{REFERENCIAS}

1. Lara A. Abián J. Alegre L, et al. Medición directa de la potencia con test de salto en voleibol femenino. Universidad de Castilla la Mancha.

2. Di Santo, M. Evaluación de la Flexibilidad. PubliCE Standard 2000; 22.

3. Arregui JA. Martínez de Haro, V. Estado actual de las investigaciones sobre la flexibilidad en la adolescencia. Revista internacional de Medicina y Ciencias de la Actividad Física y el Deporte 2001; 1 (2): 127 - 135.

4. Sale D. Postactivation potentiation: role in human performance. Exercise and Sport Sciences Reviews 2002; 30(3): 138-143.

5. Hedrick A. Entrenamiento de la flexibilidad para mejorar el rango de movimiento. Alto rendimiento 2004; 3 (16): 8-12.

6. Quintasi R. Educación Física. Aplicación de test físicos en la especialidad de Educación Física, Lima: Tarea 2006.

7. Ramos D. González JL. Mora J. (2007). Evolución de la amplitud articular en Educación Primaria y Educación Secundaria. Revista Internacional de Medicina y Ciencias de la Actividad Física y el Deporte 2007; 7 (26) 144 - 157.

8. López P., Sainz, P.; Yuste J, et al. Validez del test sit and reach unilateral como criterio de extensibilidad isquiosural. Comparación con otros protocolos. Cultura, ciencia y deporte, Año 5 2008; 8 (3) 87-92.

9. López $P$. Sáinz $P$. Rodríguez $P$, et al. A comparison of the spine posture among several sit-and-reach test protocols. Journal of Science and Medicine in Sport 2008; 10: 456462.

10. Sugiura Y. Saito T. Sakuraba K, et al. Strength déficits identified with concentric action of the hip extensors and eccentric action of the hamstrings predispose to hamstring injury in elite sprinters. Journal of orthopaedic sports physical therapy 2008; 38: 457464.

11. López P. Ferragut C. Alacid F, et al. Validez de los test dedos-planta y dedos-suelo para la valoración de la extensibilidad isquiosural en piragüistas de categoría infantil. APUNTS Medicina del deporte 2008; 157: 24-29.

12. Yeung SS, Suen AM, Yeung EW. Aprospective cohort study of hamstring injurie in competitive sprinters: preseason muscle imbalance as a possible risk factor. British Journal of Sports Medicine 2009; 43: 589- 594. 
13. Merino R. Mayorga D. Fernández E, et al. Influencia de los músculos gemelos en el test sit-and-reach tras la aplicación de kinesiotape en triatletas. Un estudio piloto. Trances 2010; 2 (6): 523-535.

14. López $P$. Alacid F. Cifosis funcional y actitud cifótica lumbar en piragüistas adolescentes. Retos 2010; 17: 5-9.

15. Chillón P. Castro J. Ruiz, JR, et al. Hip flexibility is the main determinant of the backsaver sit-and-reach test in adolescents. Journal of Sports Sciences 2010; 28 (6) 641648.

16. Heiderscheit B. Sherry M. Silder A, et al. Hamstring strain injuries: Recommendations for diagnosis, rehabilitation, and injury prevention. Journal of orthopaedic sports physical therapy 2010; 40 (2): 67-81.

17. Merino R. Mayorga D. Fernández E, et al. The effect of sole self-massage on the extensibility of the back muscle chain in triathletes. A pilot study. Journal of Sport and Health Research 2011; 3(1):17-26

18. Cohen S. Towers J. Zoga A, et al. Hamstring injuries in professional football players: magnetic resonance imaging correlation with return to play. Sports Health 2011; 3 (5) 423-430.

19. Fernández E. Merino R. Romero $O$, et al. Deportes extraescolares y flexibilidad en alumnos de primaria. TRANCES 2011; 3 (6): 695- 712.

20. Hägglund M. Walden M, Ekstrand J. Risk Factors for lower extremity muscle injury in professional soccer. The UEFA injury study. The American journal of sport medicine 2012; 41 (2): 327-335.

21. De Visser HM. Reijman M.Heijboer MP, et al. Risk factors of recurrent hamstring injuries: a systematic review. British Journal of sports medicine 2012; 46: 124-130.

22. Alonso JM. Edouard $P$. Fischetto $G$, et al. Determination of future prevention strategies in elite track and fiel: analysis of Daegu 2011 IAAF championships injuries and illnesses surveillance. British journal of sports medicine 2012; 46: 505- 514.

23. Malliaropoulos N. Mendiguchia J. Pehlivanidis $\mathrm{H}$, et al. Hamstring exercises for track and field athletes: injury and exercise biomechanics, and posible implications for exercise selection and primary prevention. British journal of sports medicine 2012; 46: 846- 851.

24. Muyor J. López $P$. Alacid F, et al. Valoración del grado de extensibilidad isquiosural en ciclistas de categoría máster 30. VIII Seminario Nacional de Nutrición, Medicina y rendimiento deportivo de Pontevedra 2012.

25. Malliaropoulos N. Non contact Hamstring injuries in sports. Muscles, Ligaments, Tendons Journal 2013; 2(4): 309-311.

26. Roberts S. Trewartha G. England M, et al. Epidemiology of time-loss injuries in English community-level rugby union. BMJ Open 2013; 3(11). 
27. Czaprowski D. Leszczewska J. Kolwicz A, et al. The comparison of the effects of three physiotherapy tchniques on hamstrings flexibility in children: a prospective, randomized, single-blind study. Plos one 2013; 8 (8).

28. Sanfilippo J. Silder A. Sherry MA, et al. Hamstring strength and morphology progression after return to sport from injury. Medicine science in sports 2013; 45 (3): 448-454.

29. Schmitt B., Tyler T. McHugh M. Rehabilitación de la Lesión Isquiotibial y prevención de la recurrencia utilizando entrenamiento excéntrico en estado alargado: Un nuevo concepto. PubliCE Standard 2013.

30. Brukner P. Nealon A. Morgan C, et al. Recurrent hamstring muscle injury:applying the limited evidence in the professional football setting with a seven-point programme. British Journal of sports medicine 2013; 48: 929-938.

31. Schmitt B. Tyler T. McHugh M. Rehabilitación de la lesión isquiotibial y prevención de la recurrencia utilizando entrenamiento excéntrico en estado alargado: Un nuevo concepto. PubliCE Standard 2013.

32. Daneshjoo A. Rahnama N. Mokhtar A, et al. Effectiveness of injury prevention programs on developing quadriceps and hamstring strength of Young male profesional soccer players. Journal of human kinetics 2013; 39: 115-125.

33. Sander A. Keiner M. Schlumberger A, et al. Effe cts of functional exercises in the warmup on sprint performances. Journal of Strength and Conditioning Research 2013; 27(4): 995-1001.

34. Padulo J. Tiloca A. Powell D, et al. EMG amplitude of the biceps femoris during jumping compared to landing movements. Springerplus 2013; 2: 520.

35. Rogan $S$. Wüst $D$. Schwitter $T$, et al. Static stretching of the hamstring muscle for injury prevention in football codes: a systematic review. Asian journal of sports medicine 2013; 4 (1): 1-9.

36. Van der Horst N. Smits D. Petersen J, et al. The preventive effect of the Nordic hamstring exercise on hamstring injuries in amateur soccer players: study protocol for a randomised controlled trial. Injury prevention 2013.

37. Daneshjoo A. Mokhtar A. Yusof A. The effects of injury prevention warm-up programmes on knee strength in male soccer players. Biology of sport 2013; 30(4): 281-288.

38. Mayorga D. Merino R. Viciana J. Criterion-Related Validity of Sit-and-Reach Tests for Estimating Hamstring and Lumbar Extensibility: a Meta-Analysis. Journal of Sports Sciences and medicine 2013; 1: 1-14.

39. Tol J. Hamilton B. Eirale C, et al. At return to play following hamstring injury the majority of professional football players have residual isokinetic deficits. British Journal of sports medicine 2014; 3. 
40. Melegati G. Tornese D. Gevi M, et al. Reducing muscle injuries and reinjuries in one italian professional male soccer team. Muscles, Ligaments, Tendons Journal 2014; 3(4): 324-330.

41. Timmins R. Opar DA. Williams MD, et al. Reduced biceps femoris myoelectrical activity influences eccentric knee flexor weakness after repeat sprint running. British Journal of sports medicine 2014; 15.

42. McCal A. Carling C. Nedelec M, et al. Risk factors, testing and preventive strategies for non-contact injuries in professional football: current perceptions and practice of 44 teams from various premier leagues. British Journal of sports medicine 2014; 16.

Número de referencias totales: 42

Número de referencias de La Revista Iberoamericana de Ciencias de la Actividad Física y del Deporte: 0 